Artículo

\title{
Respuesta agronómica de Phaseolus vulgaris a la biofertilización en campo
}

\author{
Liliana Lara-Capistrán ${ }^{1}$ \\ Luis Guillermo Hernández-Montiel ${ }^{2}$ \\ Juan José Reyes-Pérez ${ }^{3,4}$ \\ Pablo Preciado Rangel ${ }^{5}$ \\ Ramón Zulueta-Rodríguez ${ }^{1 \S}$
}

${ }^{1}$ Universidad Veracruzana-Campus Xalapa. Circuito Universitario Gonzalo Aguirre Beltrán s/n, Zona Universitaria, Xalapa, Veracruz, México. CP. 91090. (llara_capistran@hotmail.com). ${ }^{2}$ Centro de Investigaciones Biológicas del Noroeste, SC. Calle Instituto Politécnico Nacional núm. 195, Col. Playa Palo de Santa Rita Sur, La Paz, Baja California Sur, México. CP. 23096. (lhernandez@ cibnor.mx). ${ }^{3}$ Universidad Técnica de Cotopaxi. Extensión La Maná. Av. Los Almendros y Pujilí, Edificio Universitario, La Maná, Ecuador. ${ }^{4}$ Universidad Técnica Estatal de Quevedo. Av. Walter Andrade, Vía a Santo Domingo km 1.5. Quevedo, Los Ríos, Ecuador. (jjreyesp1981@gmail.com). ${ }^{5}$ Instituto Tecnológico de Torreón. Carretera Torreón-San Pedro km 7.5, Ejido Ana, Torreón, Coahuila, México. CP. 27170. (ppreciador@yahoo.com.mx).

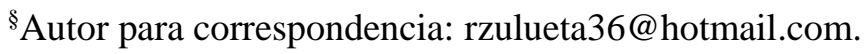

\section{Resumen}

El uso de microorganismos benéficos es una alternativa viable de incorporar en leguminosas para mejorar la fertilidad e incrementar la fijación de nitrógeno en el suelo. En este trabajo se evaluó la aplicación de hongos micorrízicos arbusculares (HMA), Rhizobium etli (Re) y una dosis reducida de fertilizante inorgánico en la producción y calidad de frijol (Phaseolus vulgaris L.) cv. 'Negro Michigan' bajo condiciones de campo. El trabajo se llevó a cabo en el Campo Experimental 'La Bandera' ubicado en el municipio de Actopan, Veracruz, durante el ciclo primavera verano 2016. Se utilizó un diseño experimental en bloques al azar con ocho tratamientos [T1: (testigo, T), T2: (fertilizado, F), T3: (inoculado con HMA), T4: (inoculado con $R e$ ), T5: (inoculado con HMA+Re), T6: (inoculado con $\mathrm{HMA}+50 \% \mathrm{~F}$ ), $\mathrm{T} 7$ : (inoculado con $R e+50 \% \mathrm{~F}$ ) y $\mathrm{T} 8$ : (inoculado con $\mathrm{HMA}+R e+50 \% \mathrm{~F})$ ], cada tratamiento con tres bloques y 500 plantas en cada uno. Se evaluó altura de la planta, diámetro del tallo, número de hojas, flores, vainas y nódulos, peso de granos, variables de calidad de las semillas, contenido de proteína total y porcentaje de colonización radicular. Se utilizó un análisis de varianza y la prueba LSD de Fisher con un nivel de significación del 5\%. Los resultados mostraron diferencias significativas entre tratamientos $(p \leq 0.05)$ para las variables registradas, siendo $\mathrm{HMA}+R e+50 \% \mathrm{~F}$ el tratamiento donde no solo se mejoró la calidad del grano de frijol, sino que el uso y los costos de fertilización se reducirían en favor de la economía de los productores.

Palabras clave: Rhizobium etli, hongos micorrízicos arbusculares, proteína.

Recibido: marzo de 2019

Aceptado: junio de 2019 


\section{Introducción}

De acuerdo con estudios de zonificación agroecológica efectuados por el INIFAP, gran proporción de la superficie destinada al cultivo del frijol en México se ubica en áreas de temporal donde el potencial agroecológico-productivo es muy vulnerable a las sequías, heladas tempranas, distribución discontinua e inestable de la precipitación pluvial durante el ciclo vegetativo de la planta o bien, al ataque de plagas y enfermedades (ASERCA, 1997; SIAP, 2000-2005) y a nivel estatal sobresale Veracruz con 1813282 ha no aptas para la siembra y cosecha de esta leguminosa (SAGARPA-INIFAP, 2012). Por consiguiente, no sorprende que durante el ciclo agrícola 2017 de otoño-invierno (humedad residual) y primavera-verano (temporal) tan solo se hayan obtenido $0.742 \mathrm{t} \mathrm{ha}^{-1}$ en la entidad veracruzana (SIAP, 2017).

No obstante, a través de la implementación de agrotecnias de vanguardia propuestas a través del mejoramiento genético (hibridación dirigida) (Muñoz, 2012) y la biotecnología es factible que los productores no solo mejoren la productividad, sino también sus condiciones de vida e ingresos (FIRA, 2016), toda vez que las distintas variedades de frijol (introducidas o criollas) podrían ocupar áreas destinadas a su cultivo sostenible con base en la tolerancia a plagas, enfermedades y estrés hídrico, o bien mediante el aprovechamiento de sus cualidades en funciones biológicas encaminadas a garantizar una auto-suficiencia alimentaria nutritiva y saludable (del Valle, 2016; Mora, 2017).

Sin embargo, diversos factores bióticos, como patógenos y virus, ocasionan considerables pérdidas económicas en las áreas de cultivo de Chiapas y Veracruz (López et al., 2007) que, aunados a la indiscriminada aplicación y encarecimiento de la arraigada fertilización nitrogenada y fosfatada requerida en la producción de este cultivo (Martínez-Viera et al., 2010; Sanchez-Yañez et al., 2014), obligan la búsqueda de alternativas capaces de resolver problemas relacionados con la seguridad alimentaria sin afectar los niveles de productividad, calidad e inocuidad deseados (Khan et al., 2016), ni dañar la fertilidad de los suelos, quebrantar la salud de los consumidores, el bienestar de los agricultores y el equilibrio ambiental (de Souza Vandenberghe et al., 2017).

Una de las opciones viables para enfrentar esta problemática radica en el uso de microorganismos cuya actividad mejore la absorción de agua y nutrimentos, disminuya la derrama de agentes contaminantes y controle la incidencia de insectos dañinos y fitopatógenos en el cultivo (AguadoSantacruz et al., 2012; Yilmaz y Sönmez, 2017). Así como, la relación simbiótica entre los organismos involucrados ocurre en la rizósfera, la finalidad perseguida a través de la investigación reside en comprobar cuales microorganismos son benéficos tras el asocio con su hospedero.

Para el caso específico del frijol, se reporta que Rhizobium etli es una rizobacteria promotora del crecimiento de leguminosas y [no leguminosas] (García-Fraile et al., 2012; Soriano y González, 2012; Shameer y Prasad, 2018) a través del mutualismo, porque además de nodular en las raíces y producir ácido acético, giberelinas y citoquininas (Mayak et al., 2004), convierte el dinitrógeno atmosférico $\left(\mathrm{N}_{2}\right)$ en ión amonio $\left(\mathrm{NH}^{4+}\right)$ (Meilhoc et al., 2011; Santi et al., 2013; Miwa y Okazaki, 2017) que fijan con gran eficiencia en el suelo (Pérez-Montaño et al., 2014) y de ahí la planta lo absorbe en las células de la raíz mediante diferentes grupos de transportadores localizados en la membrana plasmática (Kiba y Krapp, 2016). 
En cuanto a los hongos formadores de micorriza arbuscular (HMA) se refiere, Labrador (2015) y León-Aroca et al. (2017) destacan su capacidad por mantener una eficiente absorción de agua y nutrimentos, favorecer la captación de elementos primarios [especialmente fósforo] (Abd-Alla et al., 2014; Yadav et al., 2018) y otros beneficios no nutricionales imprescindibles para la supervivencia de las plantas (Halder et al., 2015; Delavaux et al., 2017) sin dejar de lado su contribución en la supresión de plagas y enfermedades (Vázquez, 2015; Jacott et al., 2017).

No obstante, el sinergismo proveniente tras la incorporación de dos microorganismos rizosféricos (Rhizobium-HMA) en el sistema radicular de leguminosas en general (Larimer et al., 2014; Pierre et al., 2014) y de frijol en particular (P. vulgaris cv. CC-25-9) (Liriano et al., 2012) ha constatado su capacidad para mejorar la sanidad y el potencial productivo de las plantas al establecer una simbiosis tripartita con innegable trascendencia ecológica (Bauer et al., 2012; Ossler et al., 2015) y agrícola (Tajini et al., 2012; Brandán de Weht et al., 2013; Martín et al., 2015).

Por tal motivo, el objetivo de este trabajo fue evaluar el efecto de la inoculación dual ( $R$. etli+HMA) y fertilización inorgánica reducida en la producción y calidad de $P$. vulgaris $\mathrm{cv}$. 'Negro Michigan' bajo condiciones de campo con el fin de conocer la factibilidad de incorporar microorganismos que coadyuven a mejorar el rendimiento y la eficiencia proteicas en estos sistemas de producción.

\section{Materiales y métodos}

\section{Localización del área de estudio}

La investigación se realizó durante el ciclo primavera-verano del 2016 en el Campo Experimental 'La Bandera', el cual pertenece a la Facultad de Ciencias Agrícolas de la Universidad Veracruzana, Campus Xalapa, ubicado en el municipio de Actopan, Veracruz, a $19^{\circ} 27^{\prime} 30^{\prime}$ ' de latitud norte, 96³4'20' de longitud oeste y altitud media de 360 msnm (Vázquez et al., 1992).

\section{Bio-insumos utilizados}

Las semillas de frijol (P. vulgaris cv. 'Negro Michigan') evaluadas fueron cosechadas en la región de Actopan, Veracruz, México y se adquirieron en las instalaciones del Campo Experimental Cotaxtla (CAECOT) del INIFAP, el cual es un órgano desconcentrado de la Secretaría de Agricultura, Ganadería, Desarrollo Rural, Pesca y Alimentación (SAGARPA). Estas se lavaron con agua e introdujeron con humedad adecuada en bolsas plásticas para agregarles el inoculante rizobacteriano ( $R$. etli) o micorrizógeno (HMA).

\section{Descripción de los tratamientos}

Los tratamientos evaluados en este trabajo fueron: T1: (testigo, T), T2: (fertilizado, F), T3: (inoculado con HMA), T4: (inoculado con $R e$ ), T5: (inoculado con HMA+Re), T6: (inoculado con $\mathrm{HMA}+50 \% \mathrm{~F}$ ), T7: (inoculado con $R e+50 \% \mathrm{~F}$ ) y T8: (inoculado con $\mathrm{HMA}+R e+50 \% \mathrm{~F}$ ). 


\section{Origen del inóculo rizobacteriano}

El inóculo bacteriano a base de $R$. etli fue proporcionado por el Laboratorio de Organismos Benéficos (LOB) de la Facultad de Ciencia Agrícolas de la Universidad Veracruzana, Campus Xalapa.

\section{Origen del inóculo micorrizógeno}

El consorcio micorrízico arbuscular RIN1-UV fue proporcionado por el LOB y estaba integrado por Rhizoglomus intraradices, Claroideoglomus etunicatum, Gigaspora albida y Glomus sp., con capacidad de colonización radicular de $\pm 85 \%$.

\section{Inoculación simple (Re) (HMA) y dual (Re+HMA)}

La inoculación simple y dual de 500 semillas de frijol cv. 'Negro Michigan' se efectuó en bolsas plásticas donde se agregaron $10 \mathrm{~mL}$ de goma arábiga como adherente, $10 \mathrm{~g}$ de inóculo micorrízico (HMA), $50 \mathrm{~g}$ de la cepa rizobacteriana ( $R e$, con turba como acarreador y concentración de $10^{5} \mathrm{UFC}$ $\left.\mathrm{g}^{-1}\right)$ y $50 \mathrm{~g} R e+10 \mathrm{~g}$ de $R e+\mathrm{HMA}$, respectivamente. Tras 5 min de agitación, las semillas se vaciaron en charolas de unicel y se secaron a la sombra para su posterior siembra.

\section{Preparación del terreno y siembra}

La unidad experimental se acondicionó mediante barbecho y rastra de un área de $93.5 \mathrm{~m}^{2}(5.5 \mathrm{~m} \mathrm{x}$ $17 \mathrm{~m}$ ), en la cual se colocaron dos semillas por golpe o postura de siembra en el camellón del surco con espeque, a $25 \mathrm{~cm}$ de distancia $\left(10\right.$ matas surco $\left.^{-1}\right)$ y $25 \mathrm{~cm}$ entre surcos. Cada parcela neta midió $3.43 \mathrm{~m}^{2}(1.25 \mathrm{~m} \times 2.75 \mathrm{~m})$.

\section{Riego}

El riego rodado a capacidad de campo se aplicó durante las etapas de la fase vegetativa y reproductiva que iniciaron tras la germinación y emergencia de las plántulas hasta antes de la floración (pre-floración) y maduración de las primeras vainas.

\section{Fertilización}

La primera aplicación de la fórmula 46-00-00 (urea 46\% N) se realizó al voleo, cuando las plántulas tenían 10 días de haber germinado y la segunda 30 días después. En los tratamientos fertilizados la aplicación se hizo al 100\% y en las dosis reducidas solo se aplicó la mitad (50\%) del fertilizante nitrogenado señalado.

\section{Diseño experimental}

El diseño experimental utilizado fue un bloques al azar con ocho tratamientos [T1: (testigo, T), T2: (fertilizado, F), T3: (inoculado con HMA), T4: (inoculado con $R e$ ), T5: (inoculado con HMA+Re), T6: (inoculado con $\mathrm{HMA}+50 \% \mathrm{~F}$ ), T7: (inoculado con $R e+50 \% \mathrm{~F}$ ) y $\mathrm{T} 8$ : (inoculado con $\mathrm{HMA}+R e+50 \% \mathrm{~F})]$ y tres repeticiones con 500 plantas en cada uno donde se midieron las siguientes variables: altura de la planta $(\mathrm{cm})$, diámetro del tallo $(\mathrm{mm})$, número de hojas, flores, vainas y 
nódulos (conteo visual), número de nódulos por planta (conteo visual), peso (g) y variables de calidad del grano (largo, ancho y grosor [mm] y espesor [g]), contenido de proteína total (\%) (AOAC, 1975) y porcentaje de colonización radicular (\%) (Giovannetti y Mosse, 1980).

\section{Clareo y tinción de raíces}

Las raíces recolectadas a los 90 días después de la siembra (DDS) se fijaron en FAA (formolácido acético y alcohol) y su clareo y tinción se realizó con la técnica sugerida por Phillips y Hayman (1970).

\section{Porcentaje de colonización radicular}

Este procedimiento se realizó por el método de la cuadrícula de Giovannetti y Mosse (1980), cuantificando la presencia de estructuras fúngicas (hifas, arbúsculos y vesículas) en las líneas verticales y horizontales de raíces colonizadas observadas con un microscopio de disección en cada tratamiento y sus respectivas repeticiones.

\section{Análisis estadístico}

Los datos obtenidos se analizaron con el software Statistica (versión 8.0. StatSoft Inc., Tulsa, USA) para Windows, y las medias fueron comparadas por la prueba de la mínima diferencia significativa LSD de Fisher con un nivel de significación de 5\% $(\alpha=0.05)$.

\section{Resultados y discusión}

El Anova reveló diferencias significativas en todas las variables evaluadas basándose en la prueba de Tukey $(p \leq 0.05)$. En altura los tratamientos $R e$ y HMA exhibieron incrementos de $140.35 \%$ y $96.85 \%$ con respecto a las plantas-testigo, mientras que la inoculación dual complementada con una dosis reducida de fertilizante inorgánico ( $\mathrm{HMA}+R e+50 \% \mathrm{~F})$ mostró mayor diámetro del tallo (176.39\%), número de hojas (201.33\%), flores (329.32\%), vainas $(312.5 \%)$ y peso de granos (620.36\%) en comparación con las plantas-testigo (Cuadro 1). Estos resultados se pueden atribuir a que los suelos donde se realizó este estudio la saturación por calcio fueron altas y el contenido de fósforo asimilable es muy bajo (8-12 ppm) debido al $\mathrm{pH}$ moderadamente alcalino (7.81-8) que le inmoviliza y no es fácilmente disponible para las plantas por encontrarse en formas insolubles (Castañeda, 2000; Zhang et al., 2017).

Cuadro 1. Comportamiento de medias para las variables evaluadas.

\begin{tabular}{lcccccccc}
\hline Tratamientos & $\begin{array}{c}\text { Altura } \\
(\mathrm{cm})\end{array}$ & $\begin{array}{c}\text { Núm. } \\
\text { hojas }\end{array}$ & \multicolumn{2}{c}{$\begin{array}{c}\text { Diámetro del Núm. de } \\
\text { tallo }(\mathrm{mm})\end{array}$} & $\begin{array}{c}\text { Núm. } \\
\text { flores }\end{array}$ & $\begin{array}{c}\text { Núm. } \\
\text { vainas }\end{array}$ & $\begin{array}{c}\text { Núm. } \\
\text { granos }\end{array}$ & $\begin{array}{c}\text { Colonización } \\
\text { nódulos }\end{array}$ \\
\hline $\mathrm{T}$ & $37.86 \mathrm{~d}$ & $15 \mathrm{e}$ & $3.22 \mathrm{~b}$ & $4.16 \mathrm{f}$ & $4.16 \mathrm{~d}$ & $4.96 \mathrm{e}$ & $5.5 \mathrm{f}$ & $3.61 \mathrm{e}$ \\
$\mathrm{F}$ & $57.76 \mathrm{c}$ & $21.66 \mathrm{de}$ & $4.7 \mathrm{~b}$ & $8 \mathrm{e}$ & $5.36 \mathrm{~d}$ & $10.2 \mathrm{~d}$ & $\mathrm{~g}$ & $2.04 \mathrm{e}$ \\
$\mathrm{HMA}$ & $74.53 \mathrm{~b}$ & $30.16 \mathrm{bc}$ & $5.71 \mathrm{ab}$ & $10.8 \mathrm{~d}$ & $11.23 \mathrm{a}$ & $14.26 \mathrm{c}$ & $9.23 \mathrm{e}$ & $86.33 \mathrm{a}$ \\
$R e$ & $91 \mathrm{a}$ & $26.6 \mathrm{~b}$ & $5.4 \mathrm{ab}$ & $9.16 \mathrm{e}$ & $7.13 \mathrm{bc}$ & $11.7 \mathrm{~cd}$ & $24.13 \mathrm{~b}$ & $13.06 \mathrm{c}$ \\
$\mathrm{HMA}+R e$ & $69.3 \mathrm{~b}$ & $31.16 \mathrm{bc}$ & $5.46 \mathrm{ab}$ & $12.86 \mathrm{c}$ & $8.4 \mathrm{bc}$ & $18.53 \mathrm{~b}$ & $27.6 \mathrm{a}$ & $85.54 \mathrm{a}$ \\
$\mathrm{HMA}+50 \% \mathrm{~F}$ & $65.8 \mathrm{bc}$ & $33.13 \mathrm{bc}$ & $6.1 \mathrm{ab}$ & $11.3 \mathrm{~d}$ & $7.66 \mathrm{bc}$ & $20.93 \mathrm{~b}$ & $12.03 \mathrm{~d}$ & $82.78 \mathrm{a}$ \\
\hline
\end{tabular}




\begin{tabular}{lcccccccc}
\hline Tratamientos & $\begin{array}{c}\text { Altura } \\
(\mathrm{cm})\end{array}$ & $\begin{array}{c}\text { Núm. } \\
\text { hojas }\end{array}$ & \multicolumn{2}{c}{$\begin{array}{c}\text { Diámetro del Núm. de } \\
\text { tallo }(\mathrm{mm})\end{array}$} & $\begin{array}{c}\text { Núm. } \\
\text { flores }\end{array}$ & $\begin{array}{c}\text { Núm. } \\
\text { vainas }\end{array}$ & $\begin{array}{c}\text { Núm. } \\
\text { granos }\end{array}$ & $\begin{array}{c}\text { Colonización } \\
\text { nódulos }\end{array}$ \\
\hline $\operatorname{Re}+50 \% \mathrm{~F}$ & $65.13 \mathrm{bc}$ & $38.93 \mathrm{~b}$ & $7.88 \mathrm{ab}$ & $14.4 \mathrm{~b}$ & $9.06 \mathrm{~b}$ & $21.23 \mathrm{~b}$ & $17.86 \mathrm{c}$ & $8.41 \mathrm{~d}$ \\
$\mathrm{HMA}+R e+50 \mathrm{~F}$ & $59.43 \mathrm{c}$ & $45.2 \mathrm{a}$ & $8.9 \mathrm{a}$ & $17.86 \mathrm{a}$ & $17.16 \mathrm{a}$ & $35.73 \mathrm{a}$ & $11.86 \mathrm{~d}$ & $82.78 \mathrm{a}$ \\
$\mathrm{SD}$ & 18 & 14 & 5 & 4 & 2 & 9.02 & 8 & 39 \\
$\mathrm{CV}$ & 28 & 47 & 92 & 37 & 36 & 52 & 66 & 87 \\
\hline
\end{tabular}

$\mathrm{SD}=$ desviación estándar; $\mathrm{CV}=$ coeficientes de variación. Los promedios con letras diferentes en la misma columna son estadísticamente diferentes (Tukey, $p \leq 0.05$ ).

Aunque se ha evidenciado que los HMA y algunos géneros bacterianos tienen la propiedad de solubilizar fosfatos aprovechables para las plantas (Martínez et al., 2013; Beltrán-Pineda, 2014; Sawers et al., 2017) y que la función predominante de los primeros es la absorción de fósforo (Wilson et al., 2012; Smith y Smith, 2012; Sarabia et al., 2017) y en los segundos la consecuente nodulación y fijación biológica de nitrógeno disponible tanto para hospederas leguminosas como no leguminosas (Meng et al., 2015), diferentes autores mencionan adaptaciones naturales atribuibles a las relaciones simbióticas con estos microorganismos donde el contenido o la disponibilidad de estos elementos es pobre o marginal (Rosas et al., 1998; Sarabia et al., 2010; Rodríguez-López et al., 2015; Zhang et al., 2016).

En este caso, la mayoría de las variables de respuesta registradas indican que el mejor tratamiento fue la interacción entre $\mathrm{HMA}+R e+50 \% \mathrm{~F}$, lo cual no solo concuerda con los beneficios reportados por Romero-García et al. (2016) en cuanto a la reducción y optimización en el uso de fungicidas y fertilizantes químicos debido al efecto positivo registrado en la fenología y biomasa de $P$. vulgaris, ya que Sánchez-Yañez et al. (2014) probaron un inoculante comercial compuesto por diversos microorganismos, entre los que destacan los HMA y las bacterias fijadoras de nitrógeno atmosférico, y evidenciaron que cuando se reduce la aplicación de $\mathrm{P}(50 \%$ fertilización nitrogenada y fosfatada, FNP) bajo un sistema de agricultura protegida, el cultivo del frijol es factible en sanidad y rendimiento con el apoyo de un inoculante mixto (p. ej. Endospore $33^{\circledR}$ ).

Por tanto, consignan justo recomendar este insumo biológico a agricultores para optimizar la dosis de FNP sin riesgo de afectar el ciclo vegetativo del frijol, la calidad del grano, ni rendimiento.

En relación con la calidad del frijol, el Anova (LSD de Fisher, $p \leq 0.05$ ) mostró diferencias significativas entre los tratamientos evaluados, siendo en el tratamiento $8(\mathrm{HMA}+\mathrm{Re}+50 \% \mathrm{~F})$ donde el contenido de proteína total fue mayor (Cuadro 2). En cuanto a las variables peso de semillas (100 unidades), espesor, ancho y largo se refiere, los mejores tratamientos fueron donde se efectuó la inoculación simple (solo HMA o Re) (Cuadro 2).

Cuadro 2. Análisis de calidad de la semilla de frijol.

\begin{tabular}{cccccc}
\hline Tratamientos & $\begin{array}{c}\text { Peso de } 100 \\
\text { semillas }(\mathrm{g})\end{array}$ & $\begin{array}{c}\text { Espesor } \\
(\mathrm{mm})\end{array}$ & Ancho $(\mathrm{mm})$ & $\begin{array}{c}\text { Largo } \\
(\mathrm{mm})\end{array}$ & $\begin{array}{c}\text { Proteína total } \\
(\%)\end{array}$ \\
\hline Testigo & $10.82 \mathrm{e}$ & $3.3 \mathrm{e}$ & $2.44 \mathrm{~d}$ & $6.29 \mathrm{~d}$ & $15.17 \mathrm{~d}$ \\
HMA & $15.06 \mathrm{a}$ & $6.51 \mathrm{a}$ & $6.58 \mathrm{a}$ & $10.21 \mathrm{a}$ & $17.88 \mathrm{~cd}$ \\
$\operatorname{Re}$ & $13.34 \mathrm{~b}$ & $5.7 \mathrm{a}$ & $6.59 \mathrm{a}$ & $10.37 \mathrm{a}$ & $19.22 \mathrm{bc}$ \\
\hline
\end{tabular}




\begin{tabular}{cccccc}
\hline Tratamientos & $\begin{array}{c}\text { Peso de 100 } \\
\text { semillas }(\mathrm{g})\end{array}$ & $\begin{array}{c}\text { Espesor } \\
(\mathrm{mm})\end{array}$ & Ancho $(\mathrm{mm})$ & $\begin{array}{c}\text { Largo } \\
(\mathrm{mm})\end{array}$ & $\begin{array}{c}\text { Proteína total } \\
(\%)\end{array}$ \\
\hline $\mathrm{F}$ & $11.55 \mathrm{de}$ & $4.15 \mathrm{~d}$ & $5.3 \mathrm{c}$ & $8.24 \mathrm{c}$ & $16 \mathrm{~d}$ \\
$\mathrm{HMA}+R e$ & $13.03 \mathrm{bc}$ & $4.77 \mathrm{c}$ & $6.41 \mathrm{ab}$ & $9.94 \mathrm{ab}$ & $20.88 \mathrm{bc}$ \\
$\mathrm{HMA}+50 \% \mathrm{~F}$ & $12.88 \mathrm{bc}$ & $4.72 \mathrm{c}$ & $6.43 \mathrm{ab}$ & $10.01 \mathrm{ab}$ & $23.99 \mathrm{~b}$ \\
$\operatorname{Re}+50 \% \mathrm{~F}$ & $12.05 \mathrm{~cd}$ & $4.64 \mathrm{~cd}$ & $6.57 \mathrm{ab}$ & $10.14 \mathrm{ab}$ & $23.89 \mathrm{~b}$ \\
$\mathrm{HMA}+R e+50 \% \mathrm{~F}$ & $12.41 \mathrm{bcd}$ & $4.67 \mathrm{c}$ & $6.24 \mathrm{~b}$ & $9.6 \mathrm{~b}$ & $25.5 \mathrm{a}$ \\
$\mathrm{SD}$ & 1.31 & 0.94 & 1.37 & 1.37 & 3.94 \\
$\mathrm{CV}$ & 10 & 19 & 23 & 14 & 19.36 \\
\hline
\end{tabular}

$\mathrm{SD}=$ desviación estándar; $\mathrm{CV}=$ coeficiente de variación. Los promedios con letras diferentes en la misma columna son estadísticamente diferentes (Tukey, $p \leq 0.05$ ).

Los rendimientos más satisfactorios se obtuvieron con el tratamiento $\mathrm{HMA}+\mathrm{Re}+50 \% \mathrm{~F}$ y en cuanto a la calidad de semillas se refiere (peso, espesor, largo y ancho) los mejores tratamientos fueron cuando las plantas se inocularon con HMA o bien con Rhizobium.

Finalmente, cabe destacar que con la interacción sinérgica entre estos microorganismos y el aporte reducido de fertilizante del tratamiento $\mathrm{HMA}+R e+50 \% \mathrm{~F}$ se obtuvieron los porcentajes más altos de proteína (25\%), respuesta que de acuerdo con Ojeda et al. (2014) parece justificable, si se toma en consideración que los HMA mejoran la absorción de fósforo mediante su red hifal, mientras que Rhizobium (bacteria simbiótica y específica de las leguminosas) requiere una demanda alta de este elemento para la fijación biológica del nitrógeno, el cual es tomado por las plantas y transformado a proteínas.

De igual manera, concuerda con lo reportado por Vargas-Torres et al. (2004) para distintos cultivares de frijol negro (Tacana, Huasteco, TLP19 y Veracruz) en los que han encontrado valores entre 18.9 y $24.2 \%$ o de $23.41 \pm 0.16 \%$ en Mayocoba (Carmona-García et al., 2007), los cuales coinciden con la cantidad y rango de proteínas que se indican en el Cuadro 2 (entre 15.17 y $25.5 \%$ ).

Sin duda, esta cualidad nutricional, aunada al aporte de fibra dietética, minerales (calcio, hierro, fósforo, magnesio y zinc) y vitaminas (tiamina, niacina y ácido fólico) en la dieta (Ulloa et al., 2011), es trascendente ya que dicha legumbre ocupa un lugar muy importante como fuente de alimentación mundial y salud humana (Pujolà et al., 2007).

\section{Colonización microbiana}

El mayor número de nódulos (X-barra 27.6 nódulos planta ${ }^{-1}$ ) fue en el tratamiento HMA+Re (Cuadro 1), matizando su ausencia en el tratamiento $F$ y que su cuantía en el tratamiento $T$ fue baja (X-barra 5-6 planta ${ }^{-1}$ ). Por otro lado, las raíces de las plantas-testigo presentaron colonización micorrízica nativa (3.61\%), las del tratamiento 3 (HMA) $86.33 \%$ y, en los restantes, estas fueron de $85.54 \%(\mathrm{HMA}+\mathrm{Re}$ ) y $82.78 \%$ (en $\mathrm{HMA}+50 \% \mathrm{~F}$ y $\mathrm{HMA}+R e+50 \% \mathrm{~F}$ ) (Cuadro 1). En cuanto a estas dos variables se refiere, dichos porcentajes fueron altos en los tratamientos donde ambos microorganismos rizosféricos (HMA y $R e$ ) interactuaron con las plantas de frijol, lo cual puede atribuirse a la sinergia proveniente de la simbiosis tripartita HMA-bacterias-leguminosa donde, tal 
y como en la literatura consultada se reporta, la colonización micorrizógena optimiza la absorción de fósforo y esta impulsa la nodulación bacteriana y fijación de nitrógeno (Rabie et al., 2005; Mortimer et al., 2008; Javaid, 2010).

Aunque en el tratamiento T las plantas de frijol presentaron bajos niveles de colonización radicular con los HMA nativos, su eficiencia no produjo los alcances agronómicos esperados. Luego entonces, el funcionamiento de la simbiosis tripartita y adaptación a las condiciones donde se estableció este agrosistema puede mejorarse mediante la inoculación con cepas previamente seleccionadas, tal como Bouizgarne et al. (2015); Miransari (2017) lo sugieren. La misma tendencia se observó en la formación de nódulos por parte de las rizobias del suelo, las cuales tampoco evidenciaron efectos benéficos en sus hospederas.

\section{Conclusiones}

En las condiciones bajo las cuales se llevó a cabo el experimento, el mejor tratamiento fue HMA+Rhizobium $+50 \% \mathrm{~F}$ donde su interacción simbiótica mejoró la calidad del grano de $P$. vulgaris cv. 'Negro Michigan', de modo tal que este manejo puede ser una alternativa viable para sustituir o al menos reducir el uso de fertilizantes inorgánicos y de esta manera, minimizar los costos a los productores agrícolas en la región de Actopan.

\section{Literatura citada}

Abd-Alla, M. H.; El-Enany, A.-W.; Nafady, N. A.; Khalaf, D. M. and Morsy, F. M. 2014. Synergistic interaction of Rhizobium leguminosarum bv. viciae and arbuscular mycorrhizal fungi as a plant growth promoting biofertilizers for faba bean (Vicia faba L.) in alkaline soil. Microbiol. Res. 169(1):49-58.

Aguado-Santacruz, G. A.; Rascón-Cruz, Q. y Luna-Bulbarela, A. 2012. Impacto económico y ambiental del empleo de fertilizantes químicos. In: Aguado-Santacruz, G. A. (Ed.). Introducción al uso y manejo de los biofertilizantes en la agricultura. Instituto Nacional de Investigaciones Forestales, Agrícolas y Pecuarias (INIFAP)-Secretaría de Agricultura, Ganadería, Desarrollo Rural, Pesca y Alimentación (SAGARPA). México, DF. 1-22 pp.

AOAC. 1975. Association of Official Analytical Chemists. Official methods of analysis. $12^{\text {th }}$ (Ed.). AOAC, Washington, DC. 1094 p.

ASERCA. 1997. La producción del frijol en México: diversidad y libre mercado. Claridades Agropecuarias. 44:3-23.

Bauer, J.; Kleczewski, N. M.; Bever, J. D.; Clay, K. and Reynolds, H. L. 2012. Nitrogen-fixing bacteria, arbuscular mycorrhizal fungi, and the productivity and structure of prairie grassland communities. Oecologia. 170(4):1089-1098.

Beltrán-Pineda, M. E. 2014. Bacterias solubilizadoras de fosfato con potencial biofertilizante en suelos cultivados con papa (Solanum tuberosum). Agron. 22(2):7-20.

Bouizgarne, B.; Oufdou K. and Ouhdouch, Y. 2015. Actinorhizal and Rhizobial-legume symbioses for alleviation of abiotic stresses. In: Arora, N. K. (Ed.). Plant microbes symbiosis: applied facets. Springer. New Delhi. 273-295 pp.

Brandán de Weht, C. I.; Amigo, J. A. and Weht, S. 2013. Simbiosis bi y tripartitas. Huayllu-Bios. 7:39-67. 
Carmona-García, R.; Osorio-Díaz, P.; Agama-Acevedo, E.; Tovar, J. and Bello-Pérez, L. A. 2007. Composition and effect of soaking on starch digestibility of Phaseolus vulgaris L. cv 'Mayocoba'. IJFST. 42(3):296-302.

Castañeda, A. M. 2000. Cartografía detallada de suelos del Campo Experimental 'La Bandera', municipio de Actopan, Veracruz. Tesis de Maestría. Facultad de Ciencias Agrícolas de la Universidad Veracruzana, Campus Xalapa. 110 p.

de Souza Vandenberghe, L. P.; García, L. M. B.; Rodrígues, C.; Camara, M. C.; Pereira, G. V. M.; de Oliveira, J. and Soccol, C. R. 2017. Potential applications of plant probiotic microorganisms in agriculture and forestry. AIMS Microbiology. 3(3):629-648.

del Valle, R. M. C. 2016. El derecho a la propiedad intelectual y la semilla. Una aproximación al análisis. México. In: Reyna, T. T. J.; Vega, L. M. y Gordillo, O. M. (Coord.). Producción, postproducción y agrotecnias de semillas, hortalizas y frutas. Coadyuvantes en la seguridad alimentaria en México y Cuba. Universidad Nacional Autónoma de México (UNAM). México, DF. 8-24 pp.

Delavaux, C. S.; Smith-Ramesh, L. M. and Kuebbing, S. E. 2017. Beyond nutrients: a metaanalysis of the diverse effects of arbuscular mycorrhizal fungi on plants and soils. Ecology. 98(8):2111-2119.

FIRA. 2016. Fideicomisos Instituidos en Relación con la Agricultura. Panorama agroalimentario; frijol 2016. México. 36 p.

García-Fraile, P.; Carro, L.; Robledo, M.; Ramírez-Bahena, M.-H.; Flores-Félix, J.-D.; Fernández, M. T.; Mateos, P. F.; Rivas, R.; Igual, J. M.; Martínez-Molina, E.; Peix, Á. and Velázquez, E. 2012. Rhizobium promotes non-legumes growth and quality in several production steps: towards a biofertilization of edible raw vegetables healthy for humans. PloS One. 7(5):e38122. doi: 10.1371/journal.pone.0038122.

Giovannetti, M. and Mosse, B. 1980. An evaluation of techniques for measuring vesicular arbuscular mycorrhizal infection in roots. New Phytol. 84(3):489-500.

Halder, M.; Mujib, A. S. M.; Khan, M. S.; Joardar, J. C.; Akhter, S. and Dhar, P. P. 2015. Effect of arbuscular mycorrhiza fungi inoculation on growth and up take of mineral nutrition in Ipomoea aquatica. Curr. World Environ. 10(1):67-75.

Jacott, C. N.; Murray, J. D. and Ridout, C. J. 2017. Trade-offs in arbuscular mycorrhizal symbiosis: Disease resistance, growth responses and perspectives for crop breeding. Agronomy. 7(4):75. doi:10.3390/agronomy7040075.

Javaid, A. 2010. Role of arbuscular mycorrhizal fungi in nitrogen fixation in legumes. In: Khan, M. S.; Zaidi, A. and Musarrat, J. (Eds.). Microbes for legumes development. SpringerVerlag/Wein, Vienna. 409-426 pp.

Khan, I.; Tango, C. N.; Miskeen, S.; Lee, B. H. and Oh, D.-H. 2016. Hurdle technology: a novel approach for enhanced food quality and safety - A review. Food Control. 73(B):1426-1444.

Kiba, T. and Krapp, A. 2016. Plant nitrogen acquisition under low availability: Regulation of uptake and root architecture. Plant Cell Physiol. 57(4):707-714.

Labrador, J. 2015. El suelo como producto de la cooperación entre lo físico y lo orgánico. In: Liceaga, I. (coor.). Sembrando en tierra viva; manual de agroecología. Proyecto AECID, La Habana. 29-42 pp.

Larimer A. L.; Clay, K. and Bever, J. D. 2014. Synergism and context dependency of interactions between arbuscular mycorrhizal fungi and rhizobia with a prairie legume. Ecology. 95(4):1045-1054. 
León-Aroca, R.; Chávez, N. E.; Aguaguiña, M. K.; Arias, V. C. y Sosa del, C. D. 2017. Evaluación de la calidad y comportamiento poscosecha de un biofertilizante. In: Jarquín, G. R. y Huerta de la P. A. (Coord.). Agricultura sostenible como base para los agronegocios. Universidad Autónoma de San Luis Potosí, México. 290-297 pp.

Liriano, G. R.; Núñez, S. D. B. y Barceló, D. R. 2012. Efecto de la aplicación de Rhizobium y mycorriza en el crecimiento del frijol (Phaseolus vulgaris L.) variedad CC-25-9 negro. Cagricola. 39(4):17-20.

López, S. E.; Tosquy, V. O. H.; Villar, S. B.; Ugalde, A. F. J.; Cumpián, G. J. y Becerra, L. E. N. 2007. Negro Papaloapan, nuevo cultivar de frijol para las áreas tropicales de Veracruz y Chiapas, México. Agric. Téc. Méx. 33(2):197-200.

Martín, G. M.; Reyes, R. and Ramírez, J. F. 2015. Coinoculación de Canavalia ensiformis (L.) D.C. con Rhizobium y hongos micorrízicos arbusculares en dos tipos de suelos de Cuba. Cultivos Tropicales. 36(2):22-29.

Martínez, R. E.; López, G. M. G.; Ormeño, O. E. and Moles, C. (Eds.). 2013. Manual teóricopráctico: Los biofertilizantes y su uso en la agricultura. SAGARPA/COFUPRO/UNAM, México. 47 p.

Martínez-Viera, R.; Dibut, B. and Ríos, Y. 2010. Efecto de la integración de aplicaciones agrícolas de biofertilizantes y fertilizantes minerales sobre las relaciones suelo-planta. Cultivos Tropicales. 31(3):27-31.

Mayak, S.; Tirosh, T. and Glick, B. R. 2004. Plant growth-promoting bacteria confer resistance in tomato plants to salt stress. Plant Physiol. Biochem. 42(6):565-572.

Meilhoc, E.; Boscari, A.; Bruand, C.; Puppo, A. and Brouquisse, R. 2011. Nitric oxide in legumerhizobium symbiosis. Plant Sci. 181(5):573-581.

Meng, L.; Zhang, A.; Wang, F.; Han, X.; Wang, D. and Li, S. 2015. Arbuscular mycorrhizal fungi and rhizobium facilitate nitrogen uptake and transfer in soybean/maize intercropping system. Front. Plant. Sci. 6:339. doi: 10.3389/fpls.2015.00339.

Miransari, M. 2017. The interactions of soil microbes affecting stress alleviation in agroecosystems. In: Kumar, V.; Kumar, M.; Sharma, S. and Prasad, R. (eds.). Probiotics in Agroecosystems. Springer Nature. Singapore. 31-50 pp.

Miwa, H. and Okazaki, S. 2017. How effectors promote beneficial interactions. Curr. Opin. Plant Biol. 38:148-154.

Mora, A. M. A. 2017. Modelos biotecnológicos de frijol GM. http://agroavances.com/eventosdetalle.php?idEve=135/.

Mortimer, P. E.; Pérez-Fernández, M. A. and Valentine, A. J. 2008. The role of arbuscular mycorrhizal colonization in the carbon and nutrient economy of the tripartite symbiosis with nodulated Phaseolus vulgaris. Soil Biol. Biochem. 40(5):1019-1027.

Muñoz, R. C. 2012. Obtención de un híbridode frijol arbustivo para una cosecha mecanizada. Tecnología en Marcha. 25(2):21-31.

Ojeda, Q. L. J.; Herrera, P. R.; Furrazola, G. E. and Hernández, R. C. 2014. Efecto de inoculaciones conjuntas de Rhizobium-micorrizas arbusculares en Leucaena leucocephala cv: Perú. Cagricola. 41(3):17-21.

Ossler, J. N.; Zielinski, C. A. and Heath, K. D. 2015. Tripartite mutualism: Facilitation or tradeoffs between rhizobial and mycorrhizal symbionts of legume hosts. Am. J. Bot. 102(8):1331-1341.

Pérez-Montaño, F.; Alías-Villegas, C.; Bellogín, R. A.; del Cerro, P.; Espuny, M. R.; JiménezGuerrero, I.; López-Baena, F. J.; Ollero, F. J. and Cubo, T. 2014. Plant growth promotion in cereal and leguminous agricultural important plants: From microorganism capacities to crop production. Microbiol. Res. 169(5-6):325-336. 
Phillips, J. M. and Hayman, D. S. 1970. Improved procedures for clearing roots and staining parasitic and vesicular-arbuscular mycorrhizal fungi for rapid assessment of infection. Trans. Brit. Mycol. Soc. 55(1):158-161.

Pierre, M. J.; Bhople, B. S.; Kumar, A.; Erneste, H.; Emmanuel, B. and Singh, Y. N. 2014. Contribution of arbuscular mycorrhizal fungi (AM fungi) and Rhizobium inoculation on crop growth and chemical properties of rhizospheric soils in high plants. IOSR-JAVS. 7(9):45-55.

Pujolà, M.; Farreras, A. and Casañas, F. 2007. Protein and starch content of raw, soaked and cooked beans (Phaseolus vulgaris L.). Food Chem. 102(4):1034-1041.

Rabie, G. H.; Aboul-Nasr, M. B. and Al-Humiany, A. 2005. Increased salinity tolerance of cowpea plants by dual inoculation of an arbuscular mycorrhizal fungus Glomus clarum and a nitrogen-fixer Azospirillum brasilense. Mycobiology. 33(1):51-60.

Rodríguez-López, C. P.; Navarro de León, A.; Arboleda-Valencia, J.W.; Valencia-Jimenez, A. y Valle-Molinares, R.H. 2015. Hongos micorrizógenos arbusculares asociados a Zea mays L. en un agroecosistema del Atlántico, Colombia. Agron. 23(1):20-34.

Romero-García, V. E.; García-Ortiz, V. R.; Hernández-Escareño, J. J. y Sánchez-Yáñez, J. M. 2016. Respuesta de Phaseolus vulgaris a microorganismos promotores de crecimiento vegetal. Sci Agropecu. 7(3):313-319.

Rosas, J. C.; Castro, J. A.; Robleto, E. A. and Handelsman, J. 1998. A method for screening Phaseolus vulgaris L. germplasm for preferential nodulation with a selected Rhizobium etli strain. Plant Soil. 203(1):71-78.

SAGARPA-INIFAP. 2012. Potencial productivo de especies agrícolas de importancia socioeconómica en México. SAGARPA/INIFAP. México, DF. 140 p.

Sanchez-Yañez, J. M.; Barrientos, R. M. G.; Balderas, L. I.; Dasgupta-Schuber, N. and MárquezBenavides, L. 2014. Respuesta de frijol al Endospor $33^{\circledR}$ a dosis $50 \%$ de fertilizante nitrogenado/fosfatado en agricultura protegida. Sci. Agropecu. 5(2):77-83.

Santi, C.; Bogusz, D. and Franche, C. 2013. Biological fixation in non-legume plants. Ann. Bot. 111(5):743-767.

Sarabia, M.; Cornejo, P.; Azcón, R.; Carreón-Abud, Y. and Larsen, J. 2017. Mineral phosphorus fertilization modulates interactions between maize, rhizosphere yeasts and arbuscular mycorrhizal fungi. Rhizosphere. 4:89-93.

Sarabia, O. M.; Madrigal, P. R.; Martínez, T. M. and Carreón, A. Y. 2010. Plantas, hongos micorrízicos y bacterias: Su compleja red de interacciones. Biológicas. 12(1):65-71.

Sawers, R. J.; Svane, S. F.; Quan, C.; Grønlund, M.; Wozniak, B.; Gebreselassie, M. N.; GonzálezMuñoz, E.; Chávez Montes, R. A.; Baxter, I.; Goudet, J.; Jakobsen, I. and Paszkowski, U. 2017. Phosphorus acquisition efficiency in arbuscular mycorrhizal maize is correlated with the abundance of root-external hyphae and the accumulation of transcripts encoding PHT1 phosphate transporters. New Phytol. 214(2):632-643.

Shameer, S. and Prasad, T. N. V. K. V. 2018. Plant growth promoting rhizobacteria for sustainable agricultural practices with special reference to biotic and abiotic stresses. Plant Growth Regul. 84(3):603-615.

SIAP. 2000-2005. Situación actual y perspectiva de frijol en México 2000-2005. http://www.campomexicano.gob.mx/portal_siap/Integracion/estadisticaderivada/ comercioexterior/estudios/perspectivas/frijol00-05.pdf.

SIAP. 2017. Avance de siembras y cosechas; año agrícola 2017, riego+temporal. http://infosiap.siap.gob.mx/agricola_siap/avancenacionalcultivo.do. 
Smith, S. E. and Smith, F. A. 2012. Fresh perspectives on the roles of arbuscular mycorrhizal fungi in plant nutrition and growth. Mycologia. 104(1):1-13.

Soriano, B. B. y González, V. A. 2012. Efecto de la inoculación de Rhizobium etli sobre el crecimiento vegetal de páprika, Capsicum annuum var. Longum, y lechuga, Lactuca sativa. REBIOL. 32(1):31-41.

Tajini, F.; Trabelsi, M. and Drevon, J.-J. 2012. Combined inoculation with Glomus intraradices and Rhizobium tropici CIAT899 increases phosphorus use efficiency for symbiotic nitrogen fixation in common bean (Phaseolus vulgaris L.). Saudi J. Biol. Sci. 19(2):157163.

Ulloa, J. A.; Rosas, U. P.; Ramírez, R. J. C. y Ulloa, R. B. E. 2011. El frijol (Phaseolus vulgaris): su importancia nutricional y como fuente de fitoquímicos. Revista Fuente. 3(8):5-9.

Vargas-Torres, A.; Osorio-Díaz, P.; Tovar, J.; Paredes-López, O.; Ruales, J. and Bello-Pérez, L. A. 2004. Chemical composition, starch bioavailability and indigestible fraction of common beans (Phaseolus vulgaris L.). Starch 56(2):74-78.

Vázquez, M. L. L. 2015. Diseño y manejo agroecológico de sistemas de producción agropecuaria. In: Liceaga, I. (Coord.). Sembrando en tierra viva; manual de agroecología. La Habana. Proyecto AECID. 185 p.

Vázquez, T. V.; Zulueta, R. R. y Lara, M. C. 1992. Análisis de la flora de malezas del Campo Experimental La Bandera, municipio de Actopan, Ver. La Ciencia y el Hombre. 11:77-106.

Wilson, B. A. L.; Ash, G. J. and Harper, J. D. I. 2012. Arbuscular mycorrhizal fungi improve the growth and nodulation of the annual legume messina (Melilotus siculus) under saline and non-saline conditions. Crop Pasture Sci. 63(2):164-178.

Yadav, A.; Suri, V. K.; Kumar, A. and Choudhary, A. K. 2018. Effect of AM fungi and phosphorus fertilization on P-use efficiency, nutrient acquisition and root morphology in pea (Pisum sativum L.) in acid Alfisol. J. Plant Nutr. 41(6):689-701.

Yilmaz, E. and Sönmez, M. 2017. The role of organic/bio-fertilizer amendment on aggregate stability and organic carbon content in different aggregate scales. Soil Tillage Res. 168:118-124.

Zhang, B.; Bu, J. and Liang, C. 2017. Regulation of nitrogen and phosphorus absorption by plasma membrane $\mathrm{H}^{+}$-ATPase in rice roots under simulated acid rain. Int. J. Environ. Sci. Technol. 14(1):101-112.

Zhang, L.; Xu, M.; Liu, Y.; Zhang, F.; Hodge, A. and Feng, G. 2016. Carbon and phosphorus exchange may enable cooperation between an arbuscular mycorrhizal fungus and a phosphate-solubilizing bacterium. New Phytol. 210(3):1022-1032. 